PROCEEDINGS OF THE

AMERICAN MATHEMATICAL SOCIETY

Volume 130, Number 11, Pages 3409-3413

S 0002-9939(02)06447-X

Article electronically published on May 14, 2002

\title{
TREE-LIKE CONTINUA DO NOT ADMIT EXPANSIVE HOMEOMORPHISMS
}

\author{
CHRISTOPHER MOURON
}

(Communicated by Alan Dow)

\begin{abstract}
A homeomorphism $h: X \rightarrow X$ is called expansive provided that for some fixed $c>0$ and every $x, y \in X$ there exists an integer $n$, dependent only on $x$ and $y$, such that $d\left(h^{n}(x), h^{n}(y)\right)>c$. It is shown that if $X$ is a tree-like continuum, then $h$ cannot be expansive.
\end{abstract}

\section{INTRODUCTION}

A continuum is a nondegenerate compact connected metric space. A homeomorphism $h: X \rightarrow X$ is called expansive provided that for some fixed $c>0$ and every $x, y \in X$ there exists an integer $n$, dependent only on $x$ and $y$, such that $d\left(h^{n}(x), h^{n}(y)\right)>c$. Expansive homeomorphisms exhibit chaotic behavior in that no matter how close two points are, either their forward or reverse image will eventually be a certain distance apart. Plykin's attractors [4] and the dyadic solenoid 6] are examples of continua that admit expansive homeomorphisms.

If $\mathcal{U}$ is a collection of open sets, the mesh of $\mathcal{U}$ is defined as $\operatorname{mesh}(\mathcal{U})=$ $\sup \{\operatorname{diam}(U): U \in \mathcal{U}\}$. If $\mathcal{U}$ is a finite open cover of continuum $X$, then the nerve of $\mathcal{U}$ is a geometric complex $\mathcal{N}(\mathcal{U})$ which has a vertex $v_{i}$ that corresponds to each element $U_{i}$ of $\mathcal{U}$ such that $\left\langle v_{i_{1}}, v_{i_{2}}, \ldots, v_{i_{j}}\right\rangle$ is a simplex of $\mathcal{N}(\mathcal{U})$ if and only if $U_{i_{1}} \cap U_{i_{2}} \cap \ldots \cap U_{i_{j}} \neq \emptyset$. A continuum $X$ is arc-like if for every $\epsilon>0$, there exists a finite open cover $\mathcal{U}$ whose mesh is less than $\epsilon$ and whose nerve is an arc. Arc-like continua are also called chainable and snake-like continua. A continuum is tree-like if for every $\epsilon>0$, there exists a finite open cover $\mathcal{U}$ whose mesh is less than $\epsilon$ and whose nerve contains no simple closed curves (i.e. a tree-graph). An open cover whose nerve is a tree is called a tree-cover. Equivalent definitions for a tree-like continuum, $X$, are the following:

1) For every $\epsilon>0$, there is an onto map $g: X \rightarrow T$ such that $\operatorname{diam}\left(g^{-1}(y)\right)<\epsilon$ for each $y \in T$ where $T$ is a tree.

2) $X=\lim _{\longleftarrow}\left\{T_{i}, f_{i}\right\}_{i=0}^{\infty}$. Where each $T_{i}$ is a tree and each $f_{i}: T_{i+1} \longrightarrow T_{i}$ is a bonding map.

A continuum is 1-dimensional if for every $\epsilon>0$ there exists a finite open cover $\mathcal{U}$ whose mesh is less than $\epsilon$ such that for every $y \in X, y$ is in at most 2 elements

Received by the editors August 16, 2000 and, in revised form, June 21, 2001.

2000 Mathematics Subject Classification. Primary 54H20, 54F50; Secondary $54 \mathrm{E} 40$.

Key words and phrases. Expansive homeomorphism, tree-like continua.

The author is pleased to acknowledge the many useful comments and suggestions made by Charles Hagopian. 
of $\mathcal{U}$. A planar continuum $X$ is a non-separating plane continuum provided that $\mathbb{R}^{2}-X$ is connected. It is important to note that all 1-dimensional non-separating plane continua are tree-like. However, not all tree-like continua can be embedded in the plane.

In order for a homeomorphism $h$ to be expansive, $h$ must stretch subcontinua. Since compactness must be preserved, these subcontinua must either be stretched and wrapped or stretched and folded. If a continuum is tree-like, some folding must occur. In this paper, we will see that stretching and folding is not enough to produce an expansive homeomorphism since folding also pushes points closer together. However, stretching and folding is enough to produce continuum-wise expansive homeomorphisms, as there are several examples of arc-like and tree-like continua that admit continuum-wise expansive homeomorphisms [2].

In 3, Kato has shown that arc-like continua do not admit expansive homeomorphisms by first showing that the pseudo-arc does not admit an expansive homeomorphism and then by lifting a homeomorphism of an arc-like continuum to a homeomorphism of the pseudo-arc. Unfortunately, these techniques cannot be extended to tree-like continua. In F.W. Worth's Dissertation, it was shown that shift homeomorphisms from the inverse limit of tree graphs cannot be expansive homeomorphisms [7], and Kato has also shown that no hereditarily decomposable tree-like continuum can admit an expansive homeomorphism. In the sequel, these results are generalized and it is shown that no tree-like continuum can admit an expansive homeomorphism.

\section{MAIN RESULTS}

The structure of the proof is as follows:

1) For purposes of a contradiction, it is assumed that $h: X \longrightarrow X$ is an expansive homeomorphism of tree-like continuum $X$ with expansive constant $c$ and let $0<$ $\epsilon<c / 3$.

2) A nondegenerate subcontinuum $M$ is found such that $\operatorname{diam}\left(h^{i}(M)\right) \rightarrow 0$ as $i \rightarrow-\infty$.

3) For each $k$, finite sequences $\left\{a^{k}=x_{i}^{k}, \ldots, x_{i_{k}}^{k}=b^{k}\right\} \subset M$ are found such that $\mathrm{d}\left(x_{i}^{k}, x_{i+1}^{k}\right)<\delta_{k}$ and $\mathrm{d}\left(a^{k}, b^{k}\right)<\gamma_{k}$, where $\delta_{k}, \gamma_{k} \rightarrow 0$.

4) For each $k$, it is shown that there are elements $x_{\alpha}^{k}, x_{\beta}^{k} \in\left\{a^{k}=x_{i}^{k}, \ldots, x_{i_{k}}^{k}=b^{k}\right\}$ such that $\mathrm{d}\left(x_{\alpha}^{k}, x_{\beta}^{k}\right)<\gamma_{k}, \mathrm{~d}\left(h^{i}\left(x_{\alpha}^{k}\right), h^{i}\left(x_{\beta}^{k}\right)\right)<\epsilon$ for all $i \leq 0$, and there exists an integer $n, 0 \leq n \leq k$, such that $\epsilon / 3 \leq \mathrm{d}\left(h^{n}\left(x_{\alpha}^{k}\right), h^{n}\left(x_{\beta}^{k}\right)\right)<\epsilon$. Let $y_{k}=h^{n}\left(x_{\alpha}^{k}\right)$ and $z_{k}=h^{n}\left(x_{\beta}^{k}\right)$.

5) For each $k$, it is shown that $\mathrm{d}\left(y_{k}, z_{k}\right) \geq \epsilon / 3$ and $\mathrm{d}\left(h^{i}\left(y_{k}\right), h^{i}\left(z_{k}\right)\right)<\epsilon$ for all $i \leq k$.

6) Finally, it is shown that there exist limit points $y, z$ of $\left\{z_{k}\right\}_{k=1}^{\infty},\left\{y_{k}\right\}_{k=1}^{\infty}$, respectively, such that $y \neq z$ and $\mathrm{d}\left(h^{i}(y), h^{i}(z)\right)<2 \epsilon<c$ which is a contradiction.

The first proposition follows from the Simple Chain Theorem which can be found in most graduate texts such as [5].

Proposition 1. Suppose $X$ is connected and $a, b \in X$. For every $\epsilon>0$ there exists a finite sequence $\left\{x_{i}\right\}_{i=1}^{n} \subset X$ such that $x_{1}=a, x_{n}=b$, and $d\left(x_{i}, x_{i+1}\right)<\epsilon$.

The previous sequence is called a simple chain sequence from $a$ to $b$ with mesh less than $\epsilon$. The next theorem is due to Kato [2]. 
Theorem 2. Let $h$ be an expansive homeomorphism of a continuum $X$. There exists a nondegenerate subcontinuum $M$ of $X$ such that either $\lim _{n \rightarrow \infty} \operatorname{diam} h^{n}(M)=$ 0 or $\lim _{n \rightarrow-\infty} \operatorname{diam} h^{n}(M)=0$.

Proposition 3. Suppose $\mathcal{T}$ is a tree cover for continuum $X$. Let a and $b$ be points of $X$ in the same element $T_{1}$ of $\mathcal{T}$ and let $\left\{x_{i}\right\}_{i=1}^{n}$ be a simple chain sequence from a to $b$ whose mesh is less than the Lebesgue number for $\mathcal{T}$. If $x_{2}$ and $x_{n-1}$ are not in the same element of $\mathcal{T}$, then there exists a $k, 2 \leq k \leq n-1$, such that $x_{k} \in T_{1}$.

Proof. Suppose not. We may assume that $x_{2}$ and $x_{n-1}$ are not in $T_{1}$. (Otherwise we are done.) Since each $x_{i}$ and $x_{i+1}$ are in a common element of $\mathcal{T}$, let $T_{i+1}$ be an element of $\mathcal{T}$ that contains both $x_{i}$ and $x_{i+1}$. Since $x_{2}$ and $x_{n-1}$ are not in the same element of $\mathcal{T}$ we can conclude that $T_{1}, T_{2}$, and $T_{n}$ are all different. Also, since $T_{i}$ and $T_{i+1}$ both contain $x_{i}, T_{i}$ and $T_{i+1}$ must intersect. Likewise, $T_{1}$ and $T_{n}$ both contain $x_{n}$ and hence must intersect. Therefore, the sequence $\left[T_{1}, T_{2}, \ldots, T_{n}, T_{1}\right]$ must contain a circle-chain. But that contradicts the fact that $\mathcal{T}$ is a tree cover.

Next, if $h$ is a homeomorphism and $n$ a positive integer, define $\mathcal{L}(h, n, \epsilon)$ to be a number greater than 0 such that

$$
d(x, y)<\mathcal{L}(h, n, \epsilon) \text { implies } d\left(h^{i}(x), h^{i}(y)\right)<\epsilon .
$$

for all $-n \leq i \leq n$, and

$$
Q_{n}(a, b)=\max _{-n \leq i \leq 0}\left\{d\left(h^{i}(a), h^{i}(b)\right)\right\} .
$$

Notice that for $n$ fixed, $Q_{n}(a, b)$ is a metric and hence follows the triangle inequality.

Lemma 4. Suppose $h: X \longrightarrow X$ is a homeomorphism of a continuum $X$ and that $\left\{x_{i}\right\}_{i=1}^{m}$ is a simple chain sequence from a to $b$ with mesh less than $\mathcal{L}(h, n, \epsilon / 6)$. Also, suppose that $\left\{x_{i}\right\}_{i=1}^{m}$ is contained in some tree-cover $\mathcal{T}$ such that a and $b$ are in the same element $T_{1}$ of $\mathcal{T}$ and that the mesh of $\left\{x_{i}\right\}_{i=1}^{m}$ is less than the Lebesgue number of $\mathcal{T}$. If $Q_{n}(a, b) \geq \epsilon$, then there exist $x_{\alpha}, x_{\beta} \in\left\{x_{i}\right\}_{i=1}^{m}$ such that $x_{\alpha}, x_{\beta}$ are in the same link of $\mathcal{T}$ and $\epsilon / 3 \leq Q_{n}\left(x_{\alpha}, x_{\beta}\right)<\epsilon$.

Proof. Given $T \in \mathcal{T}$ and subsequence $\left\{x_{i}\right\}_{i=m_{1}}^{m_{2}}$ of $\left\{x_{i}\right\}_{i=1}^{m}$ where $x_{m_{1}}, x_{m_{2}} \in T$, define $W\left(T,\left\{x_{i}\right\}_{i=m_{1}}^{m_{2}}\right)=\left\{x_{\alpha}, x_{\beta}\right\}$ where $x_{\alpha}, x_{\beta}$ are the elements of $T \cap\left\{x_{i}\right\}_{i=m_{1}}^{m_{2}}$ such that $Q_{n}\left(x_{\alpha}, x_{\beta}\right) \geq \epsilon$ and the difference between the indices, $|\alpha-\beta|$, is minimized. Notice that $W\left(T,\left\{x_{i}\right\}_{i=m_{1}}^{m_{2}}\right)$ will often not exist. However, $W\left(T_{1},\left\{x_{i}\right\}_{i=1}^{m}\right)=$ $\left\{x_{\alpha_{1}}, x_{\beta_{1}}\right\}$ where $\alpha_{1}<\beta_{1}$ exists since $Q_{n}(a, b) \geq \epsilon$. There are 3 cases to consider:

Notice that if $Q_{n}\left(x_{\alpha_{1}+1}, x_{\beta_{1}-1}\right) \geq \epsilon$, then $x_{\alpha_{1}+1}$ and $x_{\beta_{1}-1}$ cannot both be in $T_{1}$ since $\beta_{1}-1-\left(\alpha_{1}+1\right)<\beta_{1}-\alpha_{1}$.

Case 1. $Q_{n}\left(x_{\alpha_{1}+1}, x_{\beta_{1}-1}\right)<\epsilon$ and $x_{\alpha_{1}+1}, x_{\beta_{1}-1}$ are in the same element of $\mathcal{T}$.

Since $\mathrm{d}\left(h^{i}\left(x_{\alpha_{1}}\right), h^{i}\left(x_{\alpha_{1}+1}\right)\right)<\epsilon / 6$ and $\mathrm{d}\left(h^{i}\left(x_{\beta_{1}}\right), h^{i}\left(x_{\beta_{1}-1}\right)\right)<\epsilon / 6$ for all $-n \leq$ $i \leq 0$, we have $\epsilon / 3<Q_{n}\left(x_{\alpha_{1}}, x_{\beta_{1}}\right)-\epsilon / 6-\epsilon / 6<Q_{n}\left(x_{\alpha_{1}+1}, x_{\beta_{1}-1}\right)<\epsilon$ and we are done.

Case 2. $x_{\alpha_{1}+1}$ and $x_{\beta_{1}-1}$ are not contained in the same element of $\mathcal{T}$.

Then, by Proposition 3, there exists a $k_{1}, \alpha_{1}+1 \leq k_{1} \leq \beta_{1}-1$ such that $x_{k_{1}} \in T_{1} . Q_{n}\left(x_{\alpha_{1}}, x_{k_{1}}\right)<\epsilon$ and $Q_{n}\left(x_{\beta_{1}}, x_{k_{1}}\right)<\epsilon$ since $\left|k_{1}-\alpha_{1}\right|<\left|\beta_{1}-\alpha_{1}\right|$ and $\left|k_{1}-\beta_{1}\right|<\left|\beta_{1}-\alpha_{1}\right|$. Hence, by the triangle inequality, either $\epsilon / 3<Q_{n}\left(x_{\alpha_{1}}, x_{k_{1}}\right)<\epsilon$ or $\epsilon / 3<Q_{n}\left(x_{k_{1}}, x_{\beta_{1}}\right)<\epsilon$ and we are done. The next case creates an induction argument for this lemma. 
Case 3. $\epsilon \leq Q_{n}\left(x_{\alpha_{1}+1}, x_{\beta_{1}-1}\right)$ and $x_{\alpha_{1}+1}$ and $x_{\beta_{1}-1}$ are in same element of $\mathcal{T}$, say $T_{2}$.

Then $W\left(T_{2},\left\{x_{i}\right\}_{\alpha_{1}+1}^{\beta_{1}-1}\right)=\left\{x_{\alpha_{2}}, x_{\beta_{2}}\right\}$, where $\alpha_{2}<\beta_{2}$.

Suppose $T_{2}, \ldots, T_{j}$ and $x_{\alpha_{2}}, x_{\beta_{2}}, \ldots, x_{\alpha_{j}}, x_{\beta_{j}}$ have been found, again we have 3 cases to consider:

Case 1- $j . Q_{n}\left(x_{\alpha_{j}+1}, x_{\beta_{j}-1}\right)<\epsilon$ and $x_{\alpha_{j}+1}, x_{\beta_{j}-1}$ are in the same element of $\mathcal{T}$. As in Case 1, this implies that we are done.

Case 2- $j . x_{\alpha_{j}+1}$ and $x_{\beta_{j}-1}$ are not contained in the same element of $\mathcal{T}$.

As in Case 2, this implies that we are done.

Case 3- $j . \epsilon \leq Q_{n}\left(x_{\alpha_{j}+1}, x_{\beta_{j}-1}\right)$ and $x_{\alpha_{j}+1}$ and $x_{\beta_{j}-1}$ are in the same element of $\mathcal{T}$, say $T_{j+1}$.

Then $W\left(T_{j+1},\left\{x_{i}\right\}_{i=\alpha_{j}+1}^{\beta_{j}-1}\right)=\left\{x_{\alpha_{j+1}}, x_{\beta_{j+1}}\right\}$, where $\alpha_{j+1}<\beta_{j+1}$, and the induction continues.

Eventually, the induction must stop at some $j_{1}$. Otherwise, since $\alpha_{j+1}>\alpha_{j}$ and $\beta_{j+1}<\beta_{j}$, there would be a $j_{2}$ such that $\left|\beta_{j_{2}}-\alpha_{j_{2}}\right| \leq 1$, which would in turn imply $Q_{n}\left(x_{\alpha_{j_{2}}}, x_{\beta_{j_{2}}}\right)<\epsilon / 6$ which is impossible. So if the induction stops at $j_{1}$, then Case $3-j_{1}$ cannot be satisfied. Hence either Case $1-j_{1}$ or Case $2-j_{1}$ must be satisfied and the lemma is satisfied.

Lemma 5. Let $h: X \longrightarrow X$ be a homeomorphism of a compact space onto itself. Suppose that there exist sequences $\left\{y_{i}\right\}_{i=1}^{\infty},\left\{z_{i}\right\}_{i=1}^{\infty}$ such that $d\left(h^{k}\left(y_{n}\right), h^{k}\left(z_{n}\right)\right)<\epsilon$ for all $k \leq n$. Then there exists a limit point $y$ of $\left\{y_{i}\right\}_{i=1}^{\infty}$ and a limit point $z$ of $\left\{z_{i}\right\}_{i=1}^{\infty}$ such that $d\left(h^{k}(y), h^{k}(z)\right)<2 \epsilon$ for all $k$.

Proof. Let $Y$ be the set of limit points of $\left\{y_{i}\right\}_{i=1}^{\infty}$. Pick $y$ in $Y$ and let $\left\{y_{\alpha_{i}}\right\}_{i=1}^{\infty}$ be a subseqence that converges to $y$. Let $Z_{\alpha}$ be the set of limit points of $\left\{z_{\alpha_{i}}\right\}_{i=1}^{\infty}$. Pick $z \in Z_{\alpha}$ and let $\left\{z_{\beta_{i}}\right\}_{i=1}^{\infty}$ be a subsequence of $\left\{z_{\alpha_{i}}\right\}_{i=1}^{\infty}$ that converges to $z$. Then $\left\{y_{\beta_{i}}\right\}_{i=1}^{\infty}$ is a subsequence of $\left\{y_{\alpha_{i}}\right\}_{i=1}^{\infty}$ and hence also converges to $y$.

For each positive integer $n$, there exists $m_{n} \geq n$ such that $\mathrm{d}\left(y_{\beta_{m_{n}}}, y\right)<\mathcal{L}(h, n, \epsilon / 2)$ and $\mathrm{d}\left(z_{\beta_{m_{n}}}, z\right)<\mathcal{L}(h, n, \epsilon / 2)$. Thus,

$$
\begin{aligned}
d\left(h^{k}(y), h^{k}(z)\right)<d\left(h^{k}(y), h^{k}\left(y_{\beta_{m_{n}}}\right)\right) & +d\left(h^{k}\left(y_{\beta_{m_{n}}}\right), h^{k}\left(z_{\beta_{m_{n}}}\right)\right) \\
& +d\left(h^{k}\left(z_{\beta_{m_{n}}}\right), h^{k}(z)\right)<\epsilon / 2+\epsilon+\epsilon / 2
\end{aligned}
$$

for all $-n \leq k \leq n$. Since $n$ is arbitrary, the lemma holds.

Theorem 6. Tree-like continua do not admit expansive homeomorphisms.

Proof. Suppose that $h: X \longrightarrow X$ is an expansive homeomorphism of tree-like continuum $X$ with expansive constant $c$. Let $\epsilon$ be chosen such that $0<\epsilon<c / 3$. By Theorem 2, there exists a nondegenerate subcontinuum $M$ such that either $\lim _{n \rightarrow \infty}$ $\operatorname{diam} h^{n}(M)=0$ or $\lim _{n \rightarrow-\infty} \operatorname{diam} h^{n}(M)=0$. Without loss of generality, we may assume that $\operatorname{diam}\left(h^{i}(M)\right)<\epsilon$ for all $i \leq 0$. Let $\left\{\delta_{k}\right\}_{k=1}^{\infty}$ be a sequence of positive numbers such that each $\delta_{k}<\mathcal{L}(h, k, \epsilon / 6)$. Let $\mathcal{T}_{k}$ be a tree-cover of $X$ with mesh $<\delta_{k}$. Let $A_{k}$ be any $\left|\mathcal{T}_{k}\right|+1$ elements of $M$. By the pigeon-hole principle, for each $N$, there must be at least 2 elements $a_{N}^{k}, b_{N}^{k} \in A_{k}$ such that $h^{N}\left(a_{N}^{k}\right), h^{N}\left(b_{N}^{k}\right)$ are in a common element of $\mathcal{T}_{k}$. Since $A_{k}$ is finite, we may conclude that there are two elements $a_{k}, b_{k} \in A_{k}$ and a sequence of increasing integers $\left\{N_{j}\right\}_{j=1}^{\infty}$ such that $h^{N_{j}}\left(a_{k}\right)$ and $h^{N_{j}}\left(b_{k}\right)$ are in a common element of $\mathcal{T}_{k}$ for each $j$. Also, since $h$ is expansive, there exists an integer $n_{k}$ such that $\mathrm{d}\left(h^{n_{k}}\left(a_{k}\right), h^{n_{k}}\left(b_{k}\right)\right) \geq c>\epsilon$. 
Pick $N_{j_{k}} \geq n_{k}$. By Lemma 4 , there exists $x_{\alpha}^{k}, x_{\beta}^{k} \in h^{N_{j_{k}}}(M)$ such that $\epsilon / 3 \leq$ $Q_{N_{j_{k}}}\left(x_{\alpha}^{k}, x_{\beta}^{k}\right)<\epsilon$ and $\mathrm{d}\left(x_{\alpha}^{k}, x_{\beta}^{k}\right)<\delta_{k}$. Hence, $\mathrm{d}\left(h^{i}\left(x_{\alpha}^{k}\right), h^{i}\left(x_{\beta}^{k}\right)\right)<\epsilon$ for all $i \leq k$.

Now, let $m_{k}$ be the positive integer such that $\mathrm{d}\left(h^{-m_{k}}\left(x_{\alpha}^{k}\right), h^{-m_{k}}\left(x_{\beta}^{k}\right)\right) \geq \epsilon / 3$. Let $y_{k}=h^{-m_{k}}\left(x_{\alpha}^{k}\right)$ and $z_{k}=h^{-m_{k}}\left(x_{\beta}^{k}\right)$. Then $\mathrm{d}\left(h^{i}\left(y_{k}\right), h^{i}\left(z_{k}\right)\right)<\epsilon$ for all $i<k+m_{k}$. By Lemma 5, there exist limit points $y$ of $\left\{y_{k}\right\}_{k=1}^{\infty}$ and $z$ of $\left\{z_{k}\right\}_{k=1}^{\infty}$ such that $\mathrm{d}\left(h^{i}(y), h^{i}(z)\right) \leq 2 \epsilon<c$ for all $i$. However, since $\mathrm{d}\left(y_{k}, z_{k}\right) \geq \epsilon / 3, y$ and $z$ must be distinct. Therefore, $h$ is not expansive.

A continuum is decomposable if it is the union of two of its proper subcontinuum and indecomposable otherwise. A continuum is hereditarily indecomposable if every subcontinuum is indecomposable.

Question 1 (3] $)$. Does there exist a hereditarily indecomposable continuum that admits an expansive homeomorphism?

Question 2. Does there exist a non-separating plane continuum that admits an expansive homeomorphism?

If so, then it cannot be 1-dimensional.

\section{REFERENCES}

[1] H. Kato, Expansive homeomorphisms in continuum theory, Topology Appl., Proceedings of General Topology and Geometric Topology Symposium, (eds. Y. Kodama and T. Hoshina), 45(1992), no.3, 223-243. MR 93j:54023

[2] H. Kato, Continuum-wise expansive homeomorphisms, Can. J. Math. 45(1993), no. 3, 576598. MR 94k:54065

[3] H. Kato, The nonexistence of expansive homeomorphisms of chainable continua, Fund. Math. 149(1996), no. 2, 119-126. MR 97i:54049

[4] R.V. Plykin, On the geometry of hyperbolic attractors of smooth cascades, Russian Math. Surveys 39(1974), 85-131.

[5] S. Willard, General Topology, Addison-Wesley Publishing Company, Inc., Reading, Mass., 1970. MR 41:9173

[6] R.F. Williams, A note on unstable homeomorphisms, Proc. Amer. Math. Soc. 6(1955), 308309. MR 16:846d

[7] F.W. Worth, Concerning the Expansive Property and Shift Homeomorphisms of Inverse Limits, Ph.D. Dissertation, University of Missouri-Rolla, Rolla, Missouri, 1991.

Department of Mathematical Sciences, University of Delaware, Newark, Delaware 19716

E-mail address: mouron@math.udel.edu 